\title{
Tapered Meander Line Antenna for Maximum Efficiency and Minimal Environmental Impact
}

\author{
Amir Galehdar, Student Member, IEEE, David V. Thiel, Senior Member, IEEE, and Steven G. O'Keefe, Member, IEEE
}

\begin{abstract}
The environmental impact of mass produced, disposable antennas is a major consideration in such areas as RFID, cellular telephones and keyless entry devices. The selection of environmentally benign materials and low energy manufacturing impacts the efficiency of antennas. This letter outlines a design methodology based on tapering printed meander line antennas to gain maximum efficiency within a fixed area. The strategy involves an iterative optimization technique in which the thickness of each segment is changed proportionally with the current in each segment of the line. At $860 \mathrm{MHz}$, the same efficiency can be achieved with $50 \%$ of the conductive material. Alternatively, the efficiency can be increased without increasing the volume of conducting material.
\end{abstract}

Index Terms-Antenna efficiency, meander line, radio frequency identification (RFID) antennas, sustainability, taper, wire antennas.

\section{INTRODUCTION}

$\mathbf{M}$ ASS-PRODUCED antennas are now common place in consumer items such as cellular telephones, keyless entry devices, and most recently radio frequency identification (RFID) technology for tracking and identifying objects. In the past 50 years, the RFID tag production increased to 1 billion units world wide and it is projected to reach 1 trillion by 2015 as the technology may replace bar code technology [1]. However, there are major hurdles to be overcome before RFID technologies reach this level of market penetration. These challenges include reading range ${ }^{1}$ limitations, proximity degradation of tag performance, the cost of tags, and most recently, the effect of used tags on environment [2], [3]. Different solutions have been presented for improving the antenna performance and reducing the proximity effect [4]-[9], while the issue of the environmental effect of discarded antennas has recently been discussed [3], [10].

In this letter the limitations of RFID tags were addressed by introducing the tapering method to RFID meander antennas. The tapering method is demonstrated in two different ways [11]. a) The conductive material was substantially reduced while maintaining the same performance. This decreases the manufacturing cost and reduces the environmental impact. b) Using the same amount of the material redistributed along

Manuscript received December 08, 2008; revised January 18, 2009. First published February 10, 2009; current version published May 06, 2009.

The authors are with the Centre for Wireless Monitoring and Applications, Griffith University, Nathan, QLD 4111, Australia.

Color versions of one or more of the figures in this letter are available online at http://ieeexplore.iee.org.

Digital Object Identifier 10.1109/LAWP.2009.2014887

\footnotetext{
1"Reading range can be defined as the maximum distance at which RFID reader can detect the backscattered signal from the tag [13]."
}

the length of the antenna, the performance of the antenna was enhanced through an increased read range. These design methods were tested and some optimized results are presented.

\section{THEORY}

The efficiency of an antenna depends partly on conductor losses. By changing the wire radius in the RFID meander antenna in a manner directly related to the current distribution, the diameter of each conductive segment can be modified to achieve two goals - minimal conductive loss and minimum use of conductive materials. In this letter the efficiency of a wire antenna in air was calculated using the method of moments (NEC [12]). If there are $N$ segments in the NEC model of the complete antenna and the wire segment length is small enough, the current over the length of the $i$ th segment $i_{i}$, is approximately constant. If $a_{i}$ is the radius of the $i$ th segment, and all segment lengths are equal $\left(l_{i}=l=L_{\text {tot }} / N\right)$ and the radius $\left(a_{i}=a\right)$ is unchanged throughout the structure, the power loss $P_{\text {loss }}$ can be written as [11]

$$
P_{\text {loss }}=\frac{1}{2} \sqrt{\frac{\pi f \mu_{0}}{\sigma}} \frac{l}{2 \pi a} \sum_{i=1}^{N} i_{i}^{2}
$$

where $f$ is the frequency, $\mu_{0}$ is the magnetic permeability, $L_{\text {tot }}$ is the total length of the meander, and $\sigma$ is the conductivity of the wire. The efficiency $\eta$ becomes

$$
\eta=\frac{R_{\mathrm{in}} I^{2}-\sqrt{\frac{\pi f \mu_{0}}{\sigma}} \frac{l}{2 \pi a} \sum_{i=1}^{N} i_{i}^{2}}{R_{\mathrm{in}} I^{2}}
$$

where $R_{\text {in }}$ is the real part of the input impedance (Ohmic loss and radiation resistance) and $I$ is the current at the feed point (see [11], [13] for further details of the method).

The power loss was decreased by increasing the wire diameter of each segment according to the current flowing through it. This causes a change in other antenna performance parameters such as the resonant frequency which can subsequently be corrected by changing the over-all antenna length $L_{\text {tot }}$.

Two different methods of tapering were applied.

\section{A. Method 1}

The wire radius at the feed point $a_{\text {feed }}$ was fixed. The wire radius at every other segment $a_{i}$ was decreased in proportion to the original current $i_{i}$ in that segment. While the obvious choice would be to scale the radius proportionally to the power (i.e., the current squared), this resulted in a very wide range of segment radii and the antenna is almost impossible to construct 
physically. Thus $a_{i}$ was calculated using a linear current scaling factor from

$$
a_{i}= \begin{cases}a_{\text {feed }} \frac{i_{i}}{i_{\text {feed }}}, & \text { for } a_{i} \geq 0.001 \mathrm{~mm} \\ 0.001 \mathrm{~mm}, & \text { for } a_{i} \leq 0.001 \mathrm{~mm}\end{cases}
$$

with a minimum limit to the radius. The $0.001 \mathrm{~mm}$ limit was chosen to accommodate fabrication tolerances and to prevent NEC error. It was found that most antenna characteristics remained virtually unchanged even though the amount of conductive material required was significantly reduced.

\section{B. Method 2}

An iterative approach was adopted to ensure a linear relationship between current and wire radius. A further restriction was imposed: the overall conductor volume was fixed. The current distribution was calculated. The segment radius $a_{i}$ was changed in line with the current value $i_{i}$ in the segment and the current distribution recalculated. This required rescaling the radius of every segment of the antenna after each iteration. The original copper volume $V_{0}$ was calculated from

$$
V_{0}=2 \pi a^{2} L_{\text {tot }}
$$

The conductor volume of the tapered antenna $V_{T}$ was calculated using

$$
V_{T}=\sum_{i=1}^{N} 2 \pi a_{i}^{2} l_{i}
$$

where $a_{i}$ was calculated from (3). The segment current $i_{i}$ was changed when $a_{i}$ changed. The final conductor distribution was obtained using the algorithm shown in Fig. 1. The iterative scheme was found to converge after approximately 150 iterations. The routine was terminated when the radius change was less than $0.001 \mathrm{~mm}$ for every segment. While the volume of conductive materials was unchanged the antenna performance was enhanced. These two techniques have been previously applied to a straight line dipole antenna with significant success [11].

\section{MEANDER Line Results}

As examples, two published meander line structures used in RFID were modified using these two methods. The antenna details are given in Fig. 2. The first meander line dipole antenna (M1) fits the area $40 \mathrm{~mm} \times 28.1 \mathrm{~mm}$ [4]. The second structure (M2) was created using a new optimized design which had both the highest efficiency with the smallest resonant frequency in a given area for a square grid antenna [14]. Both antennas had the same area and both resonated at $869 \mathrm{MHz}$ when the wire radius was $0.05 \mathrm{~mm}$. The total length $(L)$ of M1 and M2 was 208.6 and $182.8 \mathrm{~mm}$, respectively. The segment size used in the efficiency calculation was $1 \mathrm{~mm}$. The final segment was slightly longer so the resonant frequency was accurate. These designs do not

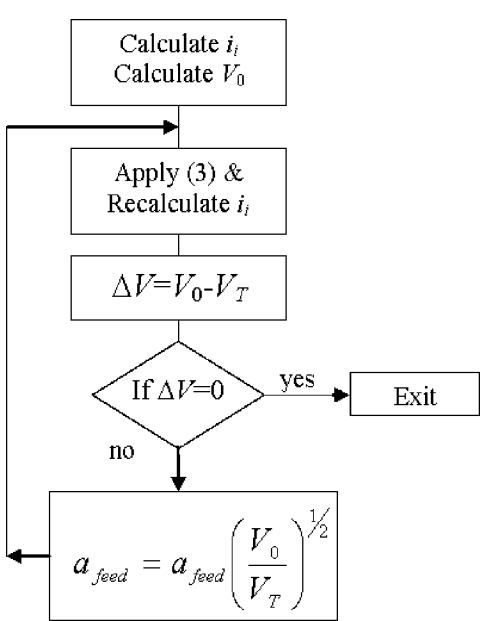

Fig. 1. Radius taper algorithm (Method 2) with fixed volume $V_{0}$.

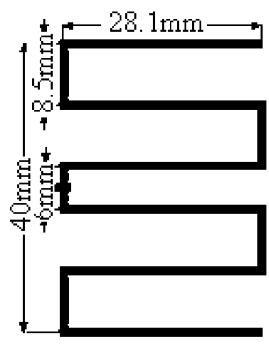

(a)

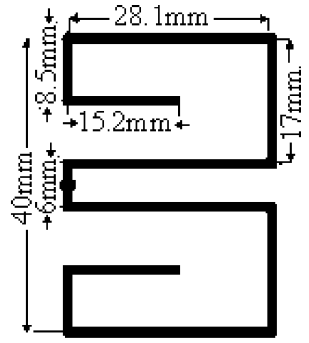

(b)
Fig. 2. Basic design parameters for the two meander line antennas (a) M1 structure for passive tags and (b) M2 structure for active tags. The wire thickness is not drawn to scale.

TABLE I

Performance Summary For ANTENNAS M1 AND M2 (SEE FIg. 2)

\begin{tabular}{|l|c|c|c|c|c|c|}
\hline Antenna & $n(\%)$ & $f_{0}(\mathrm{MHz})$ & $R_{\mathrm{a}}(\mathrm{O})$ & Total Length $(\mathrm{mm})$ & Copper Voume $\left(\mathrm{mm}^{3}\right)$ & Gain $(\mathrm{db})$ \\
\hline $\mathrm{M} 1$ & 67.2 & 869 & 8.1 & 208.2 & 1.63 & 0.177 \\
\hline $\mathrm{M} 2$ & 68.47 & 869 & 8.1 & 182.6 & 1.56 & -0.047 \\
\hline
\end{tabular}

necessarily provide the best performance in the given area. The performance published previously is summarized in Table I.

Table I indicates that these two antennas have approximately the same efficiency, input impedance and resonant frequency. M2 is preferred for an active RFID tag since the total length is shorter. M1 is preferred for passive RFID tags because it has higher gain and so has a longer read range. The effect of the changing radius was investigated in these two structures.

\section{A. Uniform Radius}

Using a uniform wire radius $a$, along the length of the dipole antenna, $a$ was changed from 0.025 to $0.1 \mathrm{~mm}$. The resonant frequency of both M1 and M2 are plotted in Fig. 3 as a function of the wire radius $a$. The resonant frequency of both antennas increases as the radius increases.

These changes can be explained in terms of the distance between some segments in the meander structure. The inductance 


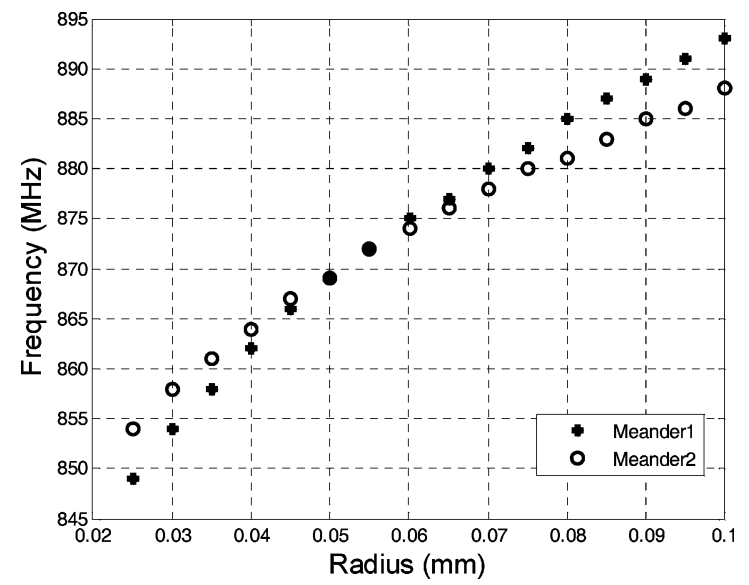

Fig. 3. Variation in resonant frequency of M1 and M2 as a function of wire radius $a$.

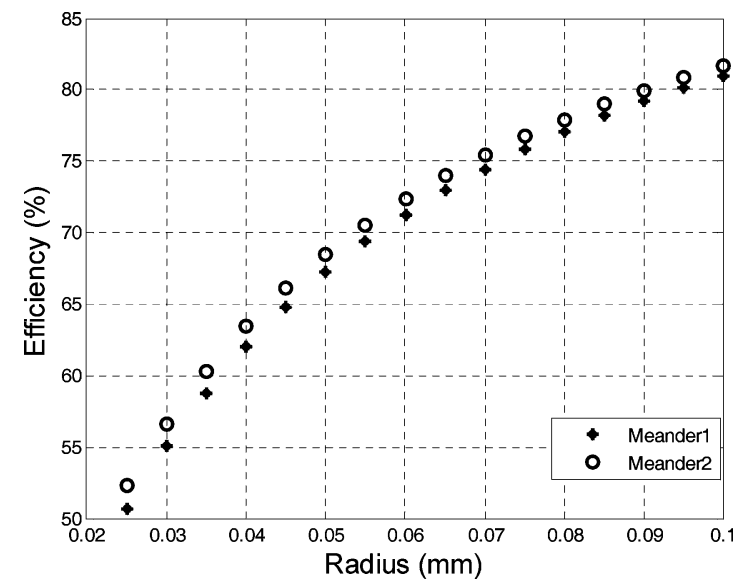

Fig. 4. Efficiency changes for M1 and M2 as a function of wire radius.

per unit length $L$ and the capacitance per unit length $C$, between two parallel wires is given by

$$
\begin{aligned}
& L=\frac{\mu}{\pi} \cosh ^{-1}\left(\frac{d}{2 a}\right) \\
& C=\frac{\pi \varepsilon}{\cosh ^{-1}\left(\frac{d}{2 a}\right)}
\end{aligned}
$$

where $d$ is the centre to centre separation and $a$ is the wire radius. Equations (6) and (7) predict that as $a$ increases, the inductance decreases and the capacitance between the segments increases. While the inductance and capacitance have opposing effects on the resonant frequency, the capacitance change dominates and the resonant frequency is increased.

In Fig. 3, it is evident that the change in resonant frequency is larger for M1 compared to M2. This implies that there is no simple mathematical expression that can be developed for this effect in meander line antennas. Fig. 4 shows the change in efficiency for the meander line antennas.

The efficiency $\eta$ of the M1 and M2 antennas increased with an increase in $a$ as indicated in (2). This is shown in Fig. 4.

M2 has a slightly higher efficiency with a shorter total length which means it is cheaper to produce and so is the better op-

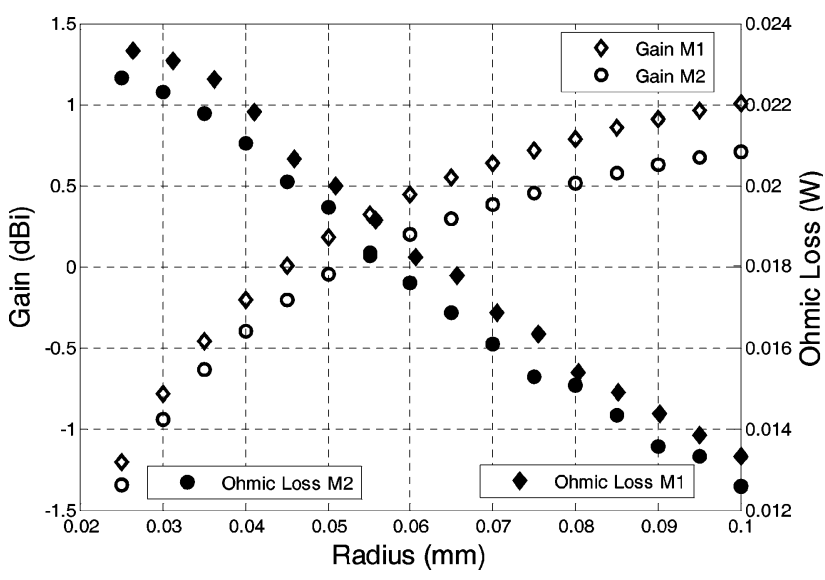

Fig. 5. Gain and Ohmic loss for M1 and M2 plotted as a function of wire radius.

TABLE II

PERFormance Summary For the Two TAPER TeCHNIQUES APPliEd to ANTENNAS M1 AND M2

\begin{tabular}{|c|c|c|c|c|c|}
\hline Antenna & $\eta(\%)$ & $f_{0}(\mathrm{MHz})$ & $R_{\text {a }}(\Omega)$ & Copper Voiume $\left(\mathrm{mm}^{3}\right)$ & Gain $(\mathrm{db})$ \\
\hline M1 & 67.2 & 869 & 8.11 & 1.63 & 0.177 \\
\hline M1-Method1 & 61.7 & 889 & 8.4 & 0.84 & 0.005 \\
\hline M1-Method2 & 69.3 & 899 & 7.68 & 1.63 & 0.55 \\
\hline M2 & 68.4 & 869 & 8.1 & 1.56 & -0.047 \\
\hline M2-Method1 & 64.7 & 890 & 8.8 & 0.81 & -0.185 \\
\hline M2-Method2 & 71.8 & 898 & 8 & 1.56 & 0.289 \\
\hline
\end{tabular}

tion for active tags. The reduction in power loss for increased radius in plotted in Fig. 5. This reduction causes an increase in efficiency and an improved gain which is important for passive tags. M1 has higher gain at low radius values and the difference increases with radius.

\section{B. Nonuniform Radius}

The two methods of tapering were applied to M1 and M2 and the results are summarized in Table II.

Taper method 1 reduced the copper volume in both structures by approximately $50 \%$ while both efficiency and gain were reduced. Taper method 2 not only improved the efficiency in both cases $(2.1 \%$ and $3.4 \%$ for $\mathrm{M} 1$ and $\mathrm{M} 2$, respectively), the gain also increased. However, the resonant frequencies of both structures increased for all cases. If the antenna is retuned, then the best structure in the given area can be determined with a minimal increase in conductor volume.

\section{MEANDER Designs}

\section{A. Minimizing Cost (Method 1)}

The cost of RFID tags is one of the boundaries that inhibits the ubiquitous use of RFID technologies. This letter presents a method of reducing the conducting area/volume (copper or silver ink) needed to manufacture RFID tags by $50 \%$. Based on these results it is possible to design an antenna with less copper without a corresponding degradation in performance. One such antenna design based on M2-Method1 is shown schematically in Fig. 6. In this antenna, the length has been increased so the antenna tuned to the same frequency as the original M2 antenna. Table III shows a 5\% loss in efficiency but the volume of copper 


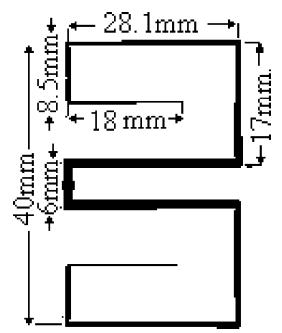

Fig. 6. Dimensions of the enhanced active RFID antenna retuned for $869 \mathrm{MHz}$.

TABLE III

PERformance Summary For ANTENNA M2 SHOWn IN Fig. 6 (EX1 Refers TO THE RETUNED ANTENNA OPTIMIZED USING METHOD 1)

\begin{tabular}{|c|c|c|c|c|c|}
\hline Antenna & $n(\%)$ & $f_{0}(\mathrm{MHz})$ & $R_{\text {in }}(\mathrm{Q})$ & Copper Volume $\left(\mathrm{mm}^{3}\right)$ & Gain $(\mathrm{db})$ \\
\hline $\mathrm{M} 2$ & 68.4 & 869 & 8.1 & 1.56 & -0.047 \\
\hline $\mathrm{M} 2-\mathrm{EX} 1$ & 62.98 & 869 & 8.59 & 0.84 & -0.331 \\
\hline
\end{tabular}

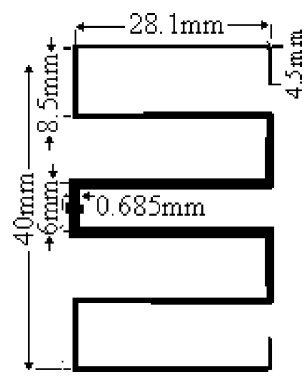

Fig. 7. The passive RFID taper antenna dimensions (wire radius is not to scale).

TABLE IV

Performance Summary for Antenna M1 Shown In Fig. 7 (EX2 Refers TO THE RETUNED ANTENNA OPTIMIZED USING METHOD 2)

\begin{tabular}{|c|c|c|c|c|c|}
\hline Antenna & $n(\%)$ & $f_{0}(\mathrm{MHz})$ & $R_{\text {ii }}(\Omega)$ & Copper Volume $\left(\mathrm{mm}^{3}\right)$ & Gain $(\mathrm{db})$ \\
\hline $\mathrm{M} 1$ & 67.2 & 869 & 8.11 & 1.63 & 0.177 \\
\hline $\mathrm{M} 1-\mathrm{E} 22$ & 67.9 & 869 & 7.74 & 1.63 & 0.431 \\
\hline
\end{tabular}

used is $50 \%$ less than that of the original meander. The tapering method has saved about $50 \%$ of the conducting material requirements.

This method can be used to reduce the manufacturing cost of RFID tags, as well as reducing the environmental impacts of disposable tags. This is an important conclusion of the letter.

\section{B. Enhancing Performance (Method 2)}

High gain tag antennas increase the read range. The tapering method can be used to create the highest gain antenna for a given volume of conducting material and area. Based on M1-Method2 model, an antenna was designed in the same area with the same volume of conductive material M1 but with higher gain (see Fig. 7 and Table IV). A thin length extension was added to decrease the resonant frequency.

This is an important conclusion from this research; that is, without any changes in cost and area, the gain of the antenna can be increased, and so the read range is larger.

\section{CONCLUSION}

In the past many antenna engineers have not paid significant attention to the environmental and construction costs of their structures. This feature has not been particularly important when manufacturing volume is low and the energy requirements in manufacture did not present a significant cost. Recently, there has been considerable emphasis on full life-cycle costing of products. With the advent of high volume manufacture of antennas in markets such as mobile telephones and RFID tags, these issues have become particularly important.

This letter has addressed three significant short comings of RFID tags which inhibit their use: expense, performance and environment effects. These problems were tackled by tapering the wire radius. The effect of changing wire radius on two meander line antennas was investigated. It was shown that an increase in the wire radius reduces the ohmic loss, and so the efficiency and gain are improved. An undesirable consequence is that the resonant frequency increased for meander antennas. It is postulated that the increase in resonant frequency for meander line antennas is due to changes in the mutual inductance and capacitance. The tapering method was applied using two different methods: a) reducing the material needed to produce antenna of similar performance, and b) enhancing the performance using the same amount of material as original antenna. These two methods were used to design better performance and/or cheaper RFID tags in given area. The technique can be applied generally to all forms of wire and printed antennas.

\section{REFERENCES}

[1] D. V. Hunt, RFID A Guide to Radio Frequency Identification. New York: Wiley, 2007.

[2] G. C. Wilshusen, "Key considerations related to federal implementation of radio frequency identification technology," in General Accounting Office Reports and Testimony, Aug. 1, 2005.

[3] D. V. Thiel, "Sustainable electronics: Wireless systems with minimal environmental impact," in Proc. ISAPE'07, Kunming, China, Nov. 2008, pp. 1298-1301.

[4] G. Marrocco, "Gain-optimized self-resonant meander line antenna for RFID application," IEEE Antenna Wireless Propag. Lett., vol. 2, pp. 302-305, 2003.

[5] D. M. Dobkin and S. M. Weigand, "Environmental effects on RFID tag antennas," in Proc. Microw. Symp. Digest, 2005 IEEE MTT-S Int., Jun. 2005, pp. 135-138.

[6] H.-W. Son and G.-Y. Choi, "Orthogonally proximity-coupled patch antenna for a passive RFID tag on metallic surfaces," Microw. Optical Technol. Lett., vol. 49, no. 3, pp. 715-717, Mar. 2007.

[7] H. Kwon and B. Lee, "Compact slotted planar inverted-F RFID tag mountable on metallic objects," Electron. Lett., vol. 41, no. 24, pp. 1308-1310, Nov. 2005.

[8] K. V. S. Rao, K. V. Nikitin, and S. F. Lam, "Antenna design for UHF RFID tags: A review and a practical application," IEEE Trans. Antennas Propag., vol. 53, no. 12, pp. 3870-3876, Dec. 2005.

[9] A. Galehdar, D. V. Thiel, and S. G. O'Keefe, "Design methods for 3D RFID antennas located on a conducting ground plane," IEEE Trans. Antennas Propag., vol. 57, no. 2, pp. 339-346, Feb. 2009.

[10] V. Subramanian, J. M. J. Frechet, P. C. Chang, D. C. Huang, J. B. Lee, A. R. Murphy, D. R. Redinger, and S. K. Volkman, "Progress toward development of all-printed RFID tags: Materials, process, and devices," IEEE Proc., vol. 93, pp. 1330-1338, Jul. 2005.

[11] A. Galehdar, D. V. Thiel, and S. G. O'Keefe, "Tapered wire antenna design for maximum efficiency and minimal environmental impact," in Proc. ISAPE'08, Kunming, China, pp. 23-26.

[12] NEC-Win Plus, User's Manual Nittany Scientific Inc, 2003.

[13] A. Galehdar, D. V. Thiel, and S. G. O'Keefe, "Antenna efficiency calculations for electrically small, RFID antennas," IEEE Antenna Wireless Propag. Lett., vol. 6, pp. 156-159, 2007.

[14] A. Galehdar, D. V. Thiel, S. G. O'Keefe, and S. P. Kingsley, "Efficiency variation in electrically small, meander line RFID antennas," in Proc. IEEE AP-S Int. Symp. Dig., Honolulu, HI, Jun. 9-15, 2007, pp. 2273-2276. 